

\title{
Dispositif élargissant une dimension seulement du faisceau de lumière issu d'un laser \\ F. Gires
}

\section{To cite this version:}

F. Gires. Dispositif élargissant une dimension seulement du faisceau de lumière issu d'un laser. Revue de Physique Appliquée, 1969, 4 (4), pp.505-506. 10.1051/rphysap:0196900404050500 jpa-00243320

\section{HAL Id: jpa-00243320 https://hal.science/jpa-00243320}

Submitted on 1 Jan 1969

HAL is a multi-disciplinary open access archive for the deposit and dissemination of scientific research documents, whether they are published or not. The documents may come from teaching and research institutions in France or abroad, or from public or private research centers.
L'archive ouverte pluridisciplinaire HAL, est destinée au dépôt et à la diffusion de documents scientifiques de niveau recherche, publiés ou non, émanant des établissements d'enseignement et de recherche français ou étrangers, des laboratoires publics ou privés. 


\title{
DISPOSITIF ÉLARGISSANT UNE DIMENSION SEULEMENT DU FAISGEAU DE LUMIÈRE ISSU D'UN LASER
}

\author{
Par F. GIRES, \\ Thomson-C.S.F., o6-Cagnes-sur-Mer. \\ (Reçu le 6 aồt 1969.)
}

\begin{abstract}
Résumé. - Un jeu de prismes permet de transformer un faisceau cohérent de section circulaire en un faisceau de section elliptique, le rapport des axes de l'ellipse étant de 10 dans la réalisation présentée.
\end{abstract}

Abstract. - A prism set transform a circular beam of light in a beam of elliptical section, the axis ratio being ten in the present result.

Les faisceaux de lumière issus du laser ont très généralement une section circulaire. Or, dans beaucoup d'applications, le traitement optique de l'information par exemple, il est nécessaire d'éclairer avec eux des objets rectangulaires dont une dimension est beaucoup plus grande que l'autre.

L'une des deux solutions suivantes est alors adoptée. $\mathrm{Ou}$ bien on utilise une paire de lentilles cylindriques et alors les aberrations distordent la surface d'onde à la sortie qui cesse d'être plane, ou bien on utilise une paire de lentilles sphériques et alors une partie plus ou moins grande de la lumière est perdue. Nous avons construit un dispositif qui fournit à partir d'une onde plane de section circulaire une onde plane de section elliptique, le rapport des axes de l'ellipse pouvant être très grand. Le rendement lumineux de l'appareil étant excellent, il est possible d'éclairer sans grandes pertes de lumière des surfaces rectangulaires de longueur égale à 10 fois la largeur.
Lorsqu'un faisceau de lumière de section circulaire tombe sur un dioptre, le faisceau émergent est elliptique. En utilisant plusieurs dioptres successifs, on peut obtenir un faisceau dont la section est aussi allongée que nécessaire.

Pour diminuer les pertes par réflexion sans traitement de surface complexe, il convient d'utiliser l'incidence brewstérienne. Dans ce cas, la transmission est unité et les largeurs des faisceaux incidents et émergents sont dans le rapport $n$, indice de réfraction du dioptre.

Afin d'obtenir avec peu de dioptres un grand rapport d'allongement, nous avons choisi un milieu de grand indice (verre E 0046 indice 1,79). L'appareil ( fig. 1) comprend un train de 4 prismes d'angle complémentaire de l'angle de Brewster. La lumière sort de chaque prisme à l'incidence normale. A la sortie de l'appareil le faisceau a une section elliptique dont le rapport des axes est $n^{4}=10,5$.

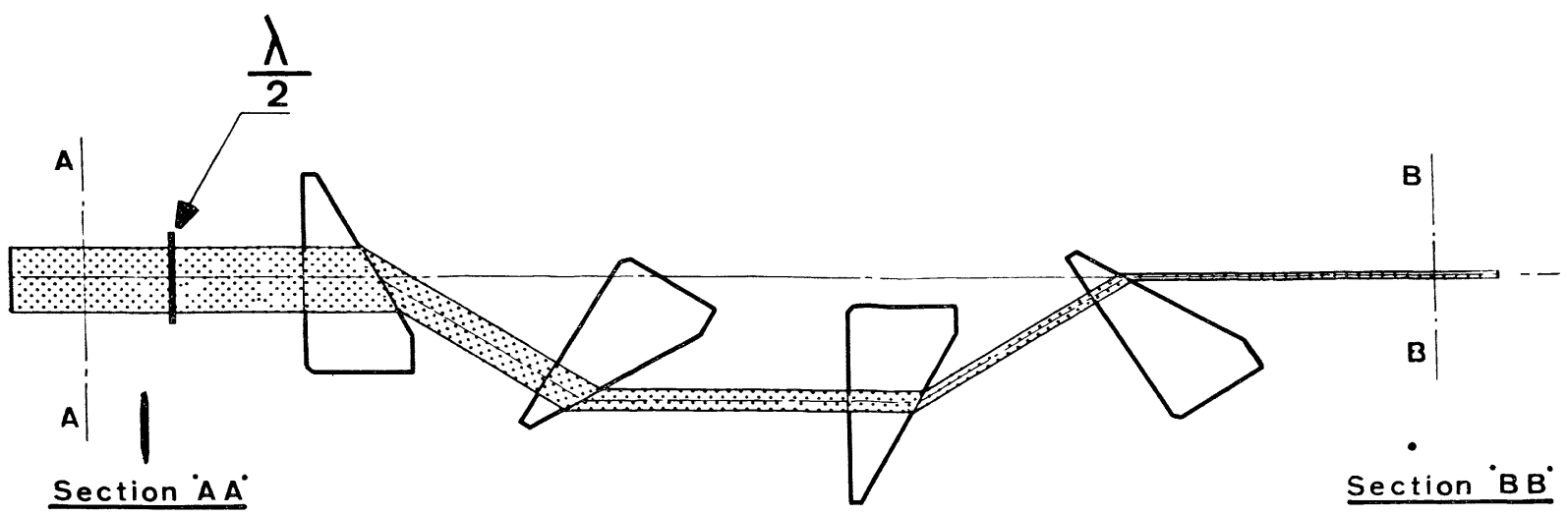

FIG. 1. 
La polarisation du faisceau incident est dans le plan d'incidence, ce qui assure une transmission d'ensemble de 0,72 assez proche de la valeur théorique $\left(\frac{4 n}{(n+1)^{2}}\right)^{4}$. Cette transmission serait proche de l'unité si les faces normales au faisceau étaient revêtues de couches diélectriques adéquates $\left({ }^{1}\right)$.

L'appareil fournissant une polarisation fixe, nous l'avons fait suivre d'une lame demi-onde qui permet, par rotation, d'obtenir n'importe quelle direction de polarisation à la sortie.

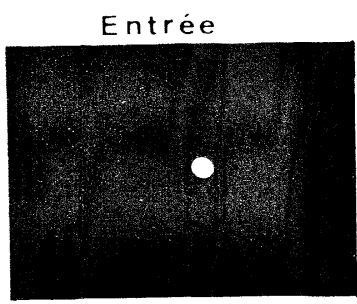

a

SECTION

La figure 2 constitue un test de la planéité de l'onde obtenue. On y voit la section du faisceau à l'entrée (a) et à la sortie (b). Une des dimensions est inchangée, l'autre est dix fois plus grande, (c) et (d) sont les figures de diffraction correspondantes. La tache d a la même longueur que la tache du faisceau incident, mais sa largeur est réduite d'un facteur de l'ordre de 10. L'onde à la sortie (b) a donc la même planéité que l'onde à l'entrée (a).

(1) Note pendant la correction des épreuves. - Après traitement des surfaces perpendiculaires au faisceau, la transmission est remontée à 0,96 .
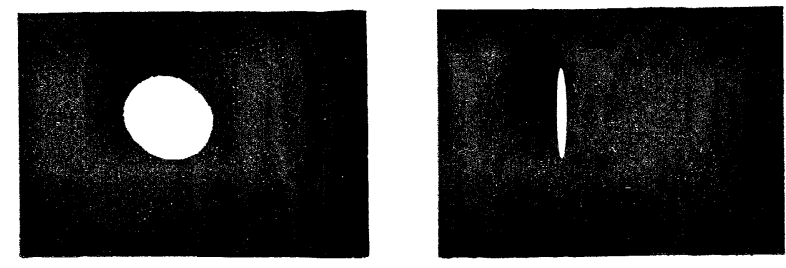

c

d

FIGURE DE DIFFRACTION

FIG. 2. 